\title{
EL OBSERVATORIO INSTITUCIONAL COMO ESTRATEGIA PARA CONTRIBUIR A LA EQUIDAD EN LA EDUCACIÓN SUPERIOR
}

\author{
O OBSERVATÓRIO INSTITUCIONAL COMO ESTRATÉGIA PARA CONTRIBUIR \\ PARA A EQUIDADE NA EDUCAÇÃO SUPERIOR
}

\author{
THE INSTITUTIONAL OBSERVATORY AS A STRATEGY TO CONTRIBUTE TO \\ EQUITY IN HIGHER EDUCATION
}

\author{
Guadalupe PALMEROS Y AVILA ${ }^{1}$ \\ Silvia Patricia AQUINO ZÚÑIGA ${ }^{2}$ \\ Verónica GARCÍA MARTÍNEZ ${ }^{3}$
}

RESUMEN: La propuesta de un Observatorio Institucional de Equidad en la Universidad Juárez Autónoma de Tabasco (UJAT), en México, es producto del proyecto de investigación Observatorio Regional para la Calidad de la Equidad en la Educación Superior (ORACLE), auspiciado por la Unión Europea a través del programa Erasmus + . En este proyecto, participan 35 Instituciones de Educación Superior de 16 países latinoamericanos y 5 europeos y entre otras cosas, propone la observación y registro sistemático de las políticas de equidad en las instituciones de educación superior. La propuesta que aquí se presenta se concibe como una estrategia para contribuir a la equidad en la educación superior. Su objetivo es la obtención de información para el análisis, seguimiento y evaluación de las políticas de equidad implementadas por la UJAT. Para ello, se aborda la concepción de la equidad, algunas políticas implementadas por la UJAT y se definen las actividades en las que el observatorio puede tener mayor incidencia.

PALABRAS CLAVE: Observatorio. Equidad. Educación superior.

RESUMO: A proposta de um Observatório da Equidade Institucional na Universidad Juárez Autónoma de Tabasco (UJAT), no México, é um produto do projeto de pesquisa Observatório Regional da Qualidade da Equidade na Educação Superior (ORACLE), patrocinado pela União Europeia para através do programa Erasmus + . Neste projeto, 35 Instituições de Ensino Superior de 16 países da América Latina e 5 países europeus participam e entre outras coisas, propõe a observação e o registro sistemático de políticas de equidade em instituições de ensino superior. A proposta apresentada aqui é concebida como uma estratégia para contribuir para a equidade no ensino superior. Seu objetivo é obter informações para a análise, monitoramento e avaliação das políticas de equidade implementadas pela UJAT. Para isso, define-se a

${ }^{1}$ Universidad Juárez Autónoma de Tabasco (UJAT), Villahermosa - Tabasco - Mexico. Profesora-investigadora del División Académica de Educación y Artes. Doctorado en Calidad y Procesos de Innovación Educativa. ORCID: http://orcid.org/0000-0001-5202-5427.E-mail: gpalmeros@hotmail.com

${ }^{2}$ Universidad Juárez Autónoma de Tabasco (UJAT), Villahermosa - Tabasco - Mexico. Profesora-investigadora del División Académica de Educación y Artes. Doctorado en Cs. de la Educación. ORCID: http://orcid.org/00000002-7223-8582. E-mail: saquinozuniga@gmail.com

${ }^{3}$ Universidad Juárez Autónoma de Tabasco (UJAT), Villahermosa - Tabasco - Mexico. División Académica de Educación y Artes. Villahermosa, Tab. México. Profesora-investigadora. Doctorado en Ciencias Sociales. ORCID: http://orcid.org/0000-0001-5299-3540.E-mail: vero1066@hotmail.com

RIAEE - Revista Ibero-Americana de Estudos em Educação, Araraquara, v. 15, n. esp. 4, p. 2587-2599, dez., 2020. e-ISSN: 1982-5587 
concepção de equidade, algumas políticas implementadas pela UJAT e as atividades em que o observatório pode ter o maior impacto.

PALAVRAS-CHAVE: Observatório. Equidade. Educação superior.

ABSTRACT: The proposal for an Institutional Observatory of Equity at the Universidad Juárez Autónoma de Tabasco (UJAT), in Mexico, is the product of the research project Regional Observatory for the Quality of Equity in Higher Education (ORACLE), sponsored by the European Union through the Erasmus + programme. In this project, 35 Higher Education Institutions from 16 Latin American and 5 European countries participate, and among other things, proposes the systematic observation and registration of equity policies in higher education institutions. The proposal presented here is conceived as a strategy to contribute to equity in higher education. Its objective is to obtain information for the analysis, monitoring and evaluation of equity policies implemented by UJAT. To this end, it addresses the concept of equity, some policies implemented by UJAT and defines the activities in which the observatory may have the greatest impact.

KEYWORDS: Observatory. Equity. Higher education.

\section{Introducción}

Sin duda alguna, la equidad en la educación es de suma importancia en el escenario actual de profundas y persistentes desigualdades sociales y educativas. Pese a las políticas, se siguen excluyendo a jóvenes en situación de vulnerabilidad a quienes no se les brinda la atención adecuada, por lo que, la discusión acerca de qué es la equidad educativa no está agotada.

La UNESCO considera que la equidad en educación implica:

Educar de acuerdo a las diferencias y necesidades individuales, sin que las condiciones económicas, demográficas, geográficas, éticas o de género supongan un impedimento al aprendizaje. Así, equidad educativa refiere a tomar en cuenta la desigual situación de los alumnos y sus familias, de las comunidades y las escuelas, ofrecer apoyos especiales a quienes lo requieren, de tal forma que los objetivos educativos sean alcanzados por el mayor número de estudiantes (UNESCO, 2002, p. 82).

Las personas de sectores desfavorables (por razones sociales, económicas, de género o de etnia) que postulan a la educación superior, son las que logran sobrevivir a una historia de desigualdades. Por ello, concretar la igualdad de oportunidades implicaría no solo facilitar el acceso a educación superior a los que logren terminar la educación media superior y obtengan un buen rendimiento en la prueba de selección, sino también, generar las condiciones para que los jóvenes puedan desarrollar efectivamente su potencial a través de toda su trayectoria.

RIAEE - Revista Ibero-Americana de Estudos em Educação, Araraquara, v. 15, n. esp. 4, p. 2587-2599, dez., 2020. e-ISSN: 1982-5587 
En las universidades, las políticas son implementadas para superar las inequidades en el acceso a los servicios educativos que ellas ofrecen, sin embargo, hay que reconocer que, a pesar de estos esfuerzos, aún quedan sin ingresar a la Educación Superior un gran porcentaje de jóvenes entre 18 y 24 años, (SEP, 2014).

Para apoyar a los estudiantes que ingresan a la Universidad, se requiere de la estructuración y diseño de mecanismos que garanticen el seguimiento, así como proveer de información que apoye a la toma de decisiones que redunden en la formulación e implementación de políticas educativas que impulsen la equidad.

Uno de estos mecanismos son los observatorios, los cuales tienen la misión de vigilar y detectar lo que ocurre en su ámbito de actuación, y su valor agregado se sustenta en: 1) buscar la información, 2) discernir su relevancia, 3) organizarla de modo coherente y 4) presentarla de forma clara (GOBIERNO VASCO, 2008).

El trabajo que aquí se presenta, tiene como objetivo dar a conocer una propuesta de estructura funcional de un Observatorio Institucional para la Calidad de la Equidad en la Universidad Juárez Autónoma de Tabasco, México que se deriva de un proyecto de investigación denominado Observatorio Regional para la Calidad de la Equidad en la Educación Superior.

\section{Políticas de equidad en la educación superior}

Las políticas de equidad en México se han convertido en una constante tanto en su normatividad como en los programas gubernamentales. Así encontramos que en el artículo tercero constitucional se reconoce la importancia de la educación y el derecho de todo individuo a recibirla (DOF, 2016). Ahora bien, en el Plan Nacional de Desarrollo 2012-2018, publicado por el (GOBIERNO DE LA REPÚBLICA, 2012, p. 125) en su meta México con educación de calidad, estableció como objetivo garantizar la inclusión y la equidad en el sistema educativo y como estrategia ampliar las oportunidades de acceso a la educación en todas las regiones y sectores de la población.

Lo anterior, implica considerar tres aspectos importantes relacionados con la equidad: El acceso efectivo, la compensación de desigualdades y la permanencia.

Si a la universidad pública le compete el compromiso de democratización social de las oportunidades de acceso, será necesario buscar esa equidad al reconocer las diferentes condiciones sociales, económicas y culturales de los aspirantes. De acuerdo con (MOKATE, 2002, p. 17): 
La igualdad de acceso representa un avance sobre el concepto de igualdad de oferta, pues no se limita a una visión de igualdad basada en la oferta, sino también se asocia con ciertos aspectos de la demanda. Garantizar la igualdad de acceso nos obliga a reconocer que los demandantes se caracterizan por condiciones muy diversas que afectan su capacidad y condición para poder responder o reaccionar a una oferta o una oportunidad y a hacer ajustes para igualar las condiciones en que diversos demandantes puedan acudir a la oportunidad que se ofrece. Estos ajustes obligan a facilitar el transporte o poner establecimientos adicionales para la entrega de servicios en áreas de baja densidad poblacional; educar e informar la demanda entre grupos que podrían faltar información o convicción con respecto al servicio o programa que se ofrece; tender a igualar los costos (monetarios y no monetarios) de acudir al servicio, etc. Igualar el acceso implica hacer que el esfuerzo relativo que el usuario tenga que hacer para aprovechar el servicio o el programa sea aproximadamente igual.

Países como México, les han apostado a políticas compensatorias sobre la base del acceso. Pero eso no es suficiente, pues hay que apoyar para garantizar la permanencia y el desarrollo de facultades o competencias adecuadas, atender a la calidad. Las políticas compensatorias deben hacerse dentro de la escuela, mediante apoyos a programas que garanticen el desarrollo de competencias, el logro de aprendizajes, el ofrecimiento de oportunidades de éxito para los estudiantes (MONZÓN 2015, p. 9), lo que nos lleva a reafirmar lo expresado por Tinto, citado por (SILVA-LAYA, 2012, p. 18) que cuando se favorece el acceso a la educación superior sin ofrecer soporte necesario a los estudiantes procedentes de entornos desfavorecidos económicamente, la puerta abierta se convierte en una puerta giratoria por la que vuelven a salir.

\section{Breves antecedentes histórico-institucionales de la evolución del tratamiento de la calidad y equidad en la Universidad Juárez Autónoma de Tabasco.}

En las últimas décadas, las instituciones de educación superior han experimentado crecientes expectativas y necesidades sentidas de parte de la sociedad alrededor de la calidad y equidad educativa, lo que les ha implicado una revisión integral de sus políticas, sobre todo, aquellas relacionadas con el acceso y equidad de los diferentes grupos sociales en lo referente a los servicios que ofrece y a la población que se dirige.

De acuerdo con la UNESCO, la educación de buena calidad, impartida por docentes competentes y bien respaldados, es un derecho de todos los niños, jóvenes y adultos, y no el privilegio de unos pocos. Señala también que la equidad, trata de

ofrecer a todos los alumnos las mejores oportunidades de alcanzar todo su potencial y de tomar medidas para abordar las situaciones de desventaja que

RIAEE - Revista Ibero-Americana de Estudos em Educação, Araraquara, v. 15, n. esp. 4, p. 2587-2599, dez., 2020. e-ISSN: 1982-5587 
limitan los logros en materia de educación. La equidad entraña un trato especial y medidas orientadas a contrarrestar las desventajas históricas y sociales que impiden que los estudiantes accedan a la educación y se beneficien de ella de manera igualitaria (UNESCO, 2015, p. 1).

Bajo este tenor, la equidad significa asegurar la igualdad de oportunidades para acceder a una educación de calidad para toda la población, proporcionando a cada quien los recursos y ayudas que requieren (UNESCO, 2007, p. 5). La falta de equidad en la Educación Superior es un problema que debe ser atendido desde los planes de desarrollo federales, estatales e institucionales que apunten decididamente a un cambio en la distribución de la población que ingresa, pero sobre todo hacia los sectores más desprotegidos. De igual forma, las condiciones para la permanencia son a su vez, otra situación que amerita mayor atención, sobre todo en los aspectos financieros, académicos y sociales, de los que se debe proveer a los estudiantes para facilitar su inclusión en el sistema, pero que además les permita culminar con éxito la fase de formación, con resultados que propicien su tránsito a la inserción productiva en la sociedad.

Ante este reto, la calidad y equidad se han convertido en una cuestión de primer orden en las agendas de muchos países, y en las instituciones educativas han adquirido un mayor protagonismo. En el caso de la UJAT, la atención a los grupos vulnerables ha cobrado mayor importancia en las primeras dos décadas de este siglo, sin embargo, ésta ha sido abordada desde una visión un tanto limitada, lo que ha resultado en políticas y estrategias débilmente articuladas entre sí o con poco seguimiento hacia los grupos vulnerables.

Por ejemplo, en el plan de desarrollo 2004-2008, el estamento al que hacía referencia es a los profesores, "Establecer redes de colaboración interdivisionales para procurar mayor integración de los cuerpos académicos que se traduzca en productividad y equidad, favoreciendo la disminución de las brechas de calidad actualmente existentes entre las Divisiones Académicas" (UJAT, 2004, p. 86).

Menciona también que "Los cuerpos colegiados deberán fortalecerse y promover dentro del ámbito de sus competencias, una Institución con identidad propia que, además de formar integralmente a sus alumnos y hacer aportaciones relevantes al medio social, respete la diversidad y promueva la equidad (UJAT, 2004, p. 93).

En Plan Estratégico de Desarrollo 2006-2016 (PDE) (UJAT, 2006), se sitúa a la inclusión de los sectores marginados como parte de las políticas de Difusión Cultural y Extensión Universitaria, pero en sus retos se señala únicamente a la educación para adultos mayores (UJAT, 2006).

En el período 2008-2012, en el Plan de Desarrollo señalaba que, 
Aparejada con la pertinencia, la respuesta que la universidad dé a las necesidades y expectativas sociales debe tener la cualidad de una búsqueda irrestricta de equidad, que significa crear ambientes bajo las cuales la universidad entiende el derecho de acceso a la educación, independientemente de la condición y circunstancias de los demandantes, y la integrada a la comunidad institucional, es decir, brindar acceso igualitario a un aprendizaje común y una formación bajo las mismas condiciones y con los mismos apoyos para su desarrollo y desempeño (UJAT, 2008, p. 85).

Continuando con esta temática, en el Plan de Desarrollo 2012-2016, se mencionaba que, a pesar de lo realizado, aún queda mucho por hacer, ya que:

se requiere reforzar el Programa del Cuidado de la Salud, Educación Sexual y Prevención de Adicciones en las 11 Divisiones Académicas; ampliar la cobertura de los apoyos a los estudiantes con dificultades económicas, fortalecer los consultorios psicopedagógicos; aumentar el índice de titulación, egreso y retención; capacitarlos en los temas de equidad de género, formación valorar, cuidado del medio ambiente y sustentabilidad; fomentar el amor al deporte, a la cultura y las artes e inculcarles, incluso a los egresados, una actitud emprendedora (UJAT, 2012, p. 68).

En este mismo plan se señalan también los siguientes aspectos en relación con la equidad y la calidad:

- Se refleja la urgencia de la Universidad de ampliar el acceso a la educación superior con equidad, pertinencia y manteniendo los niveles de calidad alcanzados.

- Ampliar la cobertura educativa, con pertinencia y equidad, creando nuevos espacios escolarizados y fortaleciendo el Sistema de Universidad Abierta y a Distancia (p. 88).

- Se garantiza ampliar la cobertura con pertinencia, calidad y equidad a través del fortalecimiento de la oferta educativa, la enseñanza a través de las tecnologías de la información, así como la apertura de nuevos espacios para dar respuesta a la demanda de los jóvenes tabasqueños y de la región de tener acceso a la Educación Superior (p. 107).

- Se ampliará la oferta de Programas Educativos de la Universidad en sus distintas modalidades y niveles con pertinencia y equidad (p. 107).

- Llevar a cabo, a través de las Comisiones Divisionales, estudios de Seguimiento de Egresados y de Empleadores de todos los Programas Educativos analizando su pertinencia, calidad y equidad (p. 108).

- Impulsar el Sistema de Educación a Distancia de la Universidad para favorecer la cobertura de educación superior con equidad y pertinencia (p. 108).

- Ampliar los programas de prevención de adicciones y de equidad de género (p. 115).

- Realizar y difundir un programa de equidad de género entre los universitarios (p. 145).

En la actualidad, la Universidad con respecto a la equidad en su Plan de Desarrollo 2016-2020, señala que

Es una institución con presencia nacional e internacional, socialmente responsable e incluyente, que contribuye al desarrollo sostenible de Tabasco y México, mediante programas educativos de calidad que forman 
profesionales éticos y competitivos en el ámbito global. Se distingue por sus relevantes aportes en la transferencia de ciencia y tecnología, el respeto y promoción de la cultura y la vinculación efectiva con su entorno, en el marco de una gestión moderna que promueve los derechos humanos, la salud integral, la transparencia y rendición de cuentas (UJAT, 2016, p. 41).

Entre los compromisos de la UJAT con la calidad educativa destacan: PC1: Promover la equidad en el acceso a la educación superior (p. 54) y PC2: Priorizar el crecimiento y diversificación de la oferta de programas educativos en sus distintas modalidades y niveles con calidad, pertinencia y equidad (p. 54)

Igualmente, en el documento del Plan de Desarrollo a Largo Plazo 2028, hace referencia:

Considerando las necesidades de cada región de la entidad, esta Alma Máter facilita el acceso a los servicios de educación superior de calidad mediante la ubicación estratégica de sus 12 divisiones académicas, así como de sus dos Centros de Educación a Distancia; estos últimos posibilitan la formación profesional de quienes combinan horarios de trabajo con sus estudios, o bien problemas de traslado por tener alguna discapacidad o por radicar en zonas muy alejadas de la Institución (UJAT, 2015, p. 27).

En este plan se entiende la calidad como: "Característica que impregna toda la vida universitaria para cumplir las funciones institucionales en óptima concordancia con las necesidades y expectativas de la sociedad en cada momento histórico" (UJAT, 2015, p. 36).

A su vez, la equidad como un "principio que defiende la igualdad de derechos, bajo las mismas condiciones, independientemente del género, realidades, circunstancias, pensamiento e ideología de los individuos o grupos sociales" (UJAT, 2015, p. 36).

Dentro del ámbito de la responsabilidad social menciona que, "mantendrá su carácter autónomo, coadyuvando a consolidar el papel de la educación superior como un bien público y como factor para la promoción de la inclusión social. Con esa finalidad, continuará garantizando la equidad en el acceso, reforzando y estableciendo medidas adecuadas para eliminar la discriminación y las desigualdades que afectan a los grupos vulnerables; así también, fortaleciendo sus vínculos con el nivel de enseñanza medio superior e incrementando la cobertura conforme a la evolución de la demanda" (UJAT, 2015, p. 64) 


\section{El Observatorio Institucional de Equidad como iniciativas para contribuir a la equidad en la educación superior.}

Para la Real Academia de la Lengua, un observatorio es un "lugar o posición que sirve para hacer observaciones"; "observación” es "acción y efecto de observar"; y "observar" es "examinar atentamente, mirar con atención y recato, atisbar".

Esta definición orienta, pero resulta insuficiente para definir y modelar un observatorio para la calidad de la equidad en la educación superior. Se trata de un observatorio calificado, en primer lugar, porque sólo mira la equidad. El concepto de equidad ha variado en el tiempo desde estar asociado a la justicia que consistía en dar a cada uno lo suyo, hasta la conceptualización que se tiene en la actualidad, el cual está asociado a la igualdad, a la no discriminación y a la inclusión.

Por su parte, HUSILLOS (2006, p. 151), señala que el término observatorio es fiel a su etimología latina observare que significa examinar o estudiar con atención, advertir o darse cuenta de una cosa, hacer notar o señalar, y la definición literal describe un lugar apropiado para hacer observaciones.

Un dato muy importante que aporta este autor es que el concepto evoluciona con dos enfoques diferentes: el primero, relacionado con almacenes de información y generación de informes, y el segundo, con formas más dinámicas sustentadas en la colaboración que estimulan la comunicación y promueven la reflexión.

En este trabajo, la equidad tiene una connotación específica y singular: mira los determinantes educativos que generan las desigualdades injustas en la educación superior. No se trata de hacerle seguimiento a la educación, sino a la inequidad, esto es, a la injusticia derivada de una relación entre dos puntos. Ello, en el entendido de que existe un nexo entre educación y vulnerabilidad, y que el sistema produce y reproduce esa inequidad.

El Observatorio Institucional de Equidad de la Universidad Juárez Autónoma de Tabasco, forma parte del Observatorio Regional para la Calidad de la equidad en la Educación Superior (ORACLE) el cual se concibió como una estructura supranacional que hoy funciona con el soporte colaborativo de los observatorios institucionales de equidad de las 35 instituciones de educación superior de 5 países europeos y 15 latinoamericanos, los cuales trabajan en red y como nodos de la red global ${ }^{4}$.

4 Las Universidades Latinoamericanas: UCB-Bolivia; UMRPSXCH-Bolivia; UNESP-Brasil; UNB-Brasil; UTALCA-Chile; UT-Colombia; U de A-Colombia; ITCR-Costa Rica; UCR-Costa Rica; UCF-Cuba; UES-El Salvador; UJMD-El Salvador; ESPOL-Ecuador; UNEMI-Ecuador; URL-Guatemala; FLACSO-Guatemala; UDELAS- Panamá; UIP-Panamá; UNAN-Nicaragua, UNAN-LEÓN-Nicaragua; UV-México; UCP-Paraguay;

RIAEE - Revista Ibero-Americana de Estudos em Educação, Araraquara, v. 15, n. esp. 4, p. 2587-2599, dez., 2020. e-ISSN: 1982-5587 
Estos observatorios están constituidos como redes que cumplen diversas funciones como la vigilancia, evaluación y control de ciertos aspectos de interés público, así como el señalamiento de las áreas de oportunidad que son susceptibles de ser intervenidas o solucionadas.

En el caso del Observatorio Institucional de Equidad de la Universidad Juárez Autónoma de Tabasco tendrá la función de mirar las políticas que genere, configurando un espacio privilegiado de participación entre todos los actores, buscando fomentar cambios organizacionales para conducir a la mejora de la calidad de la equidad.

De igual forma, emitirá estudios e informes con datos obtenidos a través de experiencias previas y metodologías exitosas, con los objetivos de:

a) Diagnosticar la situación de los diferentes colectivos

b) Generar modelos que permitan evaluar la calidad y la innovación universitaria.

c) Identificar el grado de equidad con la que las instituciones de educación superior diseñan sus políticas y actuaciones.

Sobre estos objetivos, el Observatorio Institucional debe contribuir a que la UJAT sea una universidad equitativa entre todos los ciudadanos y ofrezca garantía de calidad por igual a toda la población.

El Observatorio se encuentra insertado en la UJAT de la siguiente manera:

Figura 1 - Diagrama de flujo el Observatorio Institucional

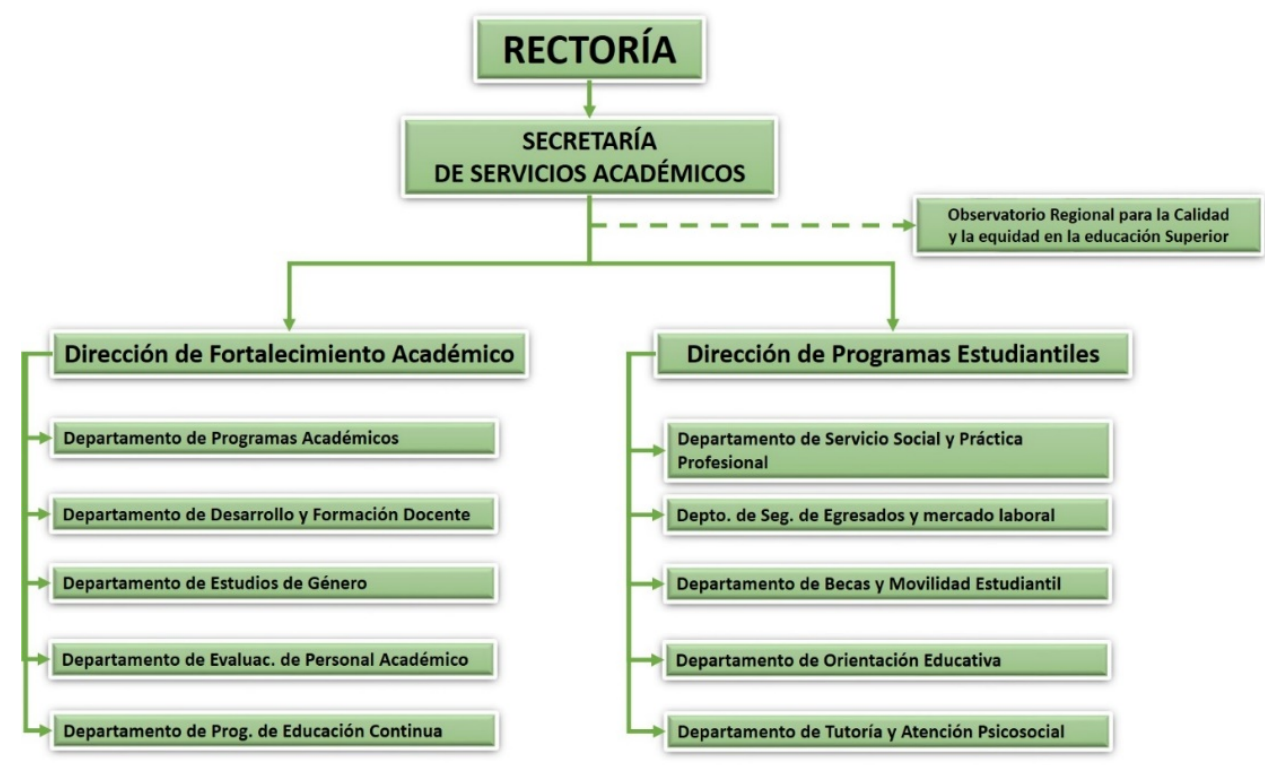

Fuente: Elaborado por los autores

NA-Paraguay, IUACJ-Uruguay; ORT-Uruguay; ULA-Venezuela; UCV-Venezuela. Las Universidades europeas: FHJ-Austria; AAU-Dinamarca, UAB-España; UNIBG-Italia; IPL-Portugal.

RIAEE - Revista Ibero-Americana de Estudos em Educação, Araraquara, v. 15, n. esp. 4, p. 2587-2599, dez., 2020. e-ISSN: 1982-5587 
Con respecto al Observatorio Institucional de Equidad (OEI) y en función del organigrama de la UJAT y las dependencias relacionadas con la calidad y equidad en los distintos estamentos, se propone la siguiente estructura:

Figura 2 - Diagrama de flujo el Consejo Universitario

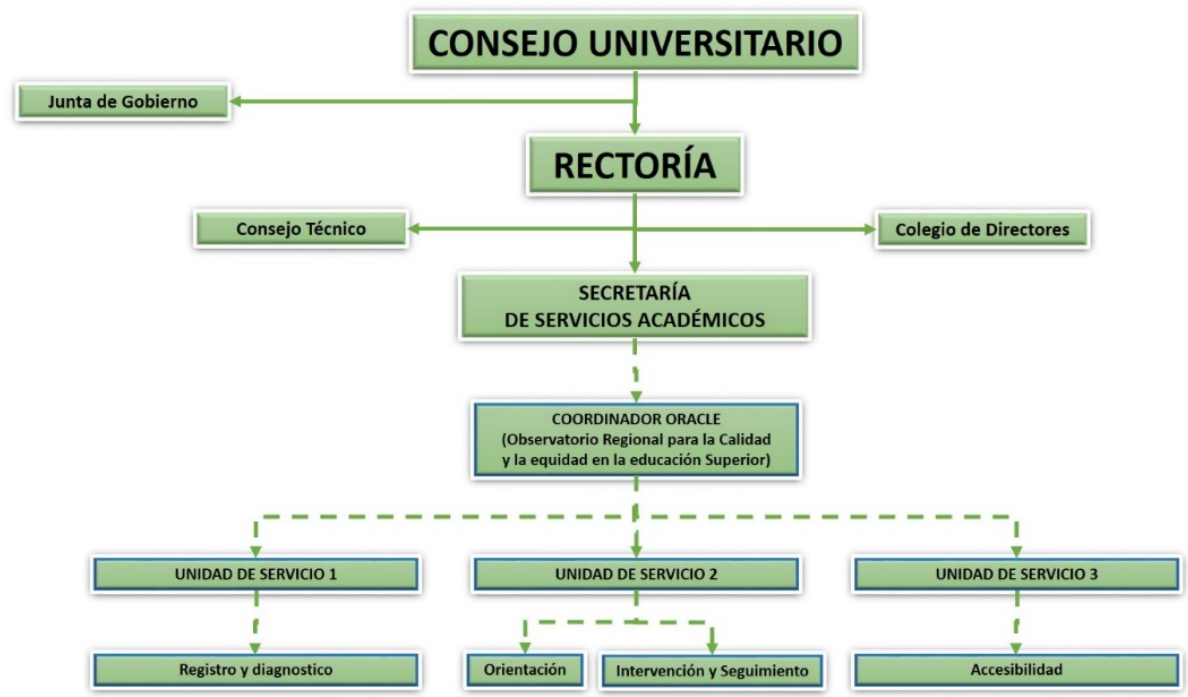

Fuente: Elaborado por los autores

A partir del observatorio, se pueden generar, políticas, normativas, planes de acción, presupuestos y cobertura para la atención de los estamentos en coordinación con las áreas señaladas, ya que, en el diagnóstico realizado en la UJAT y las evidencias que se tienen, nos demuestran que hasta el momento hay grupos a los cuales no se les ha dado atención alguna y en otros sólo se han atendido de manera, por ejemplo:

1. Igualdad de género. Existe un departamento en la universidad, se han generado políticas, normativas, planes de acción, cuenta con presupuesto y la cobertura es a nivel institucional, cubriendo todos los estamentos.

2. Alumnos con discapacidad, se atienden, pero no existen políticas, normativas ni instancias para su atención. De manera específica en la División Académica de Educación y Artes se tiene un programa, pero no hay un plan de acción, ni se cuenta con presupuesto ni registro de buenas prácticas para su atención.

3. Personal académico y administrativo con discapacidad, hasta el momento no se han generado políticas ni normativas, mucho menos instancias, planes de acción, presupuesto y cobertura. De hecho, no hay un registro de cuántos hay en la UJAT.

4. Estudiantes provenientes de grupos indígenas. Sólo se tiene información de ellos a su ingreso a la universidad, pero no hay seguimiento ni apoyos para su trayectoria académica.

5. Alumnos con IDH bajo, se tienen políticas, normativa, instancias, presupuesto y cobertura, pero al igual que las políticas anteriores, no hay un seguimiento de ellas. 


\section{Consideraciones finales}

El Observatorio de Equidad de la UJAT fue inaugurado el pasado cinco de julio de 2019 y el funcionamiento recae en los cuerpos académicos participantes en el proyecto ORACLE. Este equipo de profesores investigadores, tienen la responsabilidad de proponer, dar seguimiento, evaluar las políticas de equidad promovidas por la institución, así como realizar investigaciones, proponer cursos de formación y actualización en materia de equidad, apoyar a los profesores y estudiantes en la adaptación curricular y de materiales de estudio. La tarea no es fácil, pero se tienen algunos avances significativos al integrar la equidad de grupos vulnerables como línea de investigación emergente en el recientemente creado posgrado de Doctorado en Educación. Esta acción permitirá no sólo abonar en el campo investigativo, sino formar recursos humanos de nivel de pregrado a través de tesis, servicio social, prácticas profesionales, entre otros.

A través de este observatorio se espera incrementar indicadores de equidad de grupos vulnerables como la obtención de recursos anuales específicos para implementar políticas institucionales a favor de estos grupos, la creación de un plan de equidad de grupos vulnerables dentro de la institución, el desarrollo de un reglamento para la implementación de la equidad de grupos vulnerables que permita darle seguimiento, la conformación de una red nacional que fomente el intercambio de experiencias y enriquezca los procesos de implementación de la equidad de grupos vulnerables en las IES, así como promover vínculos con la legislatura federal y las legislaturas estatales.

Sistematizar y dar seguimiento a las sesiones de inducción, los resultados de los exámenes de admisión, estudios socioeconómicos y el análisis para caracterizar a los estudiantes de nuevo ingreso, son algunas de las estrategias de este observatorio para brindar opciones de equidad e implementar programas remediales y/o cursos propedéuticos o de nivelación que permitan que todos los alumnos tengan el perfil idóneo para iniciar sus estudios de pregrado.

Estas estrategias permitirán a los Comités Institucionales de Evaluación de la Educación Superior (CIEES) y comités acreditadores, contar con evidencias de la existencia de la equidad de los grupos vulnerables sobre su funcionamiento, cobertura, operación y resultados del observatorio.

En México, atender la equidad de grupos vulnerables representa uno de los propósitos en el ámbito educativo; la UJAT consciente de este compromiso, valora a todos los estudiantes para darles un trato que propicie superar las desigualdades sociales, que permita el desarrollo 
de una sociedad justa, equilibrada basada en los Derechos Humanos, para así potenciar las cualidades de cada ser.

\section{REFERENCIAS}

GOBIERNO DE LA REPÚBLICA. Plan Nacional de Desarrollo 2013-2018. Diario Oficial de la Federación, 2013. Disponible en: http://pnd.gob.mx/. Acceso em: 10 sep. 2020.

GOBIERNO VASCO. Observatorio Vasco de la Previsión Social. Presentación. 2008.

Disponible en: http://www.euskadi.eus/gobierno-

vasco/contenidos/informacion/presentacion_observatorio_ssc/es_9837/observatorio_ssc.html. Acceso em: 10 sep. 2020.

HUSILLOS, J. Inmigración y gobierno local. Experiencias y retos. IV Seminario Inmigración y Europa. P.149.154, 2006.

MÉXICO. Secretaría de Educación Pública. Ley General de Educación. Diario Oficial de la Federación. Última reforma DOF 22-03-2017. Disponible en:

https://www.sep.gob.mx/work/models/sep1/Resource/558c2c24-0b12-4676-ad90-

8ab78086b184/ley_general_educacion.pdf. Acceso em: 10 sep. 2020.

MÉXICO. Secretaría de Educación Pública. Principales Cifras del Sistema Educativo Nacional 2013-2014. México, 2014.

MÉXICO. Secretaría de Gobernación. Constitución Política de los Estados Unidos

Mexicanos. Diario Oficial de la Federación. Última reforma DOF 24-02-2017. Disponible en: http://dof.gob.mx/nota_detalle.php?codigo=5472965\&fecha=24/02/2017. Acceso em: 10 sep. 2020.

MOKATE, K. Eficacia, eficiencia, equidad y sostenibilidad: ¿Qué queremos decir? Banco Interamericano de Desarrollo, Instituto Interamericano para el Desarrollo Social (INDES). 2002. Disponible en

http://www.cepal.org/ilpes/noticias/paginas/9/37779/gover_2006_03_eficacia_eficiencia.pdf. Acceso em: 10 sep. 2020.

MONZÓN, M. Y. Una política educativa: la equidad. 2015. Disponible en https://www.ride.org.mx/index.php/RIDE/article/view/110/489. Acceso em: 10 sep. 2020.

SILVA-LAYA, M. Equidad en la educación superior en México: la necesidad de un nuevo concepto y nuevas políticas. Archivos Analíticos de Políticas Educativas, v. 20, n. 4, 2012. Disponible en: http://epaa.asu.edu/ojs/article/view/965. Acceso em: 10 sep. 2020.

UNESCO. Educación de Calidad para Todos. Un asunto de Derechos Humanos. París: UNESCO, 2007.

UNESCO. Foro Mundial sobre la Educación 2015. Educación de calidad, equitativa e inclusiva así como un aprendizaje durante toda la vida para todos en 2030 . Transformar 
vidas mediante la Educación. 2015. Disponible en: http://es.unesco.org/world-educationforum-2015/5-key-themes/equidad. Acceso em: 10 sep. 2020.

UNIVERSIDAD JUÁREZ AUTÓNOMA DE TABASCO. Plan Estratégico de Desarrollo 2006-2016. Disponible en: http://www.archivos.ujat.mx/rectoria/Plan2006-2016/Plan20062016.pdf. Acceso em: 10 sep. 2020.

UNIVERSIDAD JUÁREZ AUTÓNOMA DE TABASCO. Plan de Desarrollo a Largo Plazo 2028. 2015. Disponible en: http://www.ujat.mx/rectoria/7119. Acceso em: 10 sep. 2020.

UNIVERSIDAD JUÁREZ AUTÓNOMA DE TABASCO. Plan de Desarrollo institucional 2004-2008. Por una Universidad de Calidad. 2004. Disponible en:

http://www.archivos.ujat.mx/DGPEI/PDI\%202004-2008.pdf. Acceso em: 10 sep. 2020.

UNIVERSIDAD JUÁREZ AUTÓNOMA DE TABASCO. Plan de Desarrollo Institucional 2008-2012. 2008. Disponible en: http://www.archivos.ujat.mx/DGPEI/PDI\%202008-

2012.pdf. Acceso em: 10 sep. 2020.

UNIVERSIDAD JUÁREZ AUTÓNOMA DE TABASCO. Plan de Desarrollo Institucional 2012-2016. 2012. Disponible en:

http://www.archivos.ujat.mx/2012/rectoria/PLAN\%20DE\%20DESARROLLO\%20INSTITU CIONAL\%202012-2016.pdf. Acceso em: 10 sep. 2020.

UNIVERSIDAD JUÁREZ AUTÓNOMA DE TABASCO. Plan de Desarrollo Institucional 2016-2020. Disponible en: http://www.ujat.mx/rectoria/17651. Acceso em: 10 sep. 2020.

\section{Cómo referenciar este artículo}

PALMEROS Y AVILA, G.; AQUINO ZÚÑIGA, S. P.; GARCÍA MARTÍNEZ, V. El observatorio institucional como estrategia para contribuir a la equidad en la educación superior. Revista Ibero-Americana de Estudos em Educação, Araraquara, v. 15, n. esp. 4, p. 25872599, dez., 2020. e-ISSN: 1982-5587. DOI: https://doi.org/10.21723/riaee.v15iesp4.14507

Remitido el: 10/09/2019

Revisiones requeridas el: 10/01/2020

Aprobado el: $30 / 04 / 2020$

Publicado el: 01/12/2020 